\title{
Increased serum concentration of ceramides in obese children with nonalcoholic fatty liver disease
}

Natalia Wasilewska ${ }^{1 *}$, Anna Bobrus-Chociej ${ }^{1}$, Ewa Harasim-Symbor², Eugeniusz Tarasów ${ }^{3}$, Małgorzata Wojtkowska ${ }^{4}$, Adrian Chabowski ${ }^{2}$ and Dariusz M. Lebensztejn ${ }^{1}$

\begin{abstract}
Background: Hepatic lipid accumulation is closely related to the development of insulin resistance, which is regarded as one of the most significant risk factors of nonalcoholic fatty liver disease (NAFLD). Although the exact molecular pathway leading to impaired insulin signaling has not been definitively established, ceramides are suspected mediators of lipid induced hepatic insulin resistance. Therefore, the aim of the study was to evaluate the serum ceramides concentration in obese children with NAFLD.

Methods: The prospective study included 80 obese children (aged 7-17 years, median 12 years) admitted to our Department to diagnose initially suspected liver disease. Patients with viral hepatitis (HCV, HBV, CMV), autoimmune (AlH), toxic and metabolic (Wilson's disease, alfa-1-antitrypsin deficiency) liver diseases and celiac disease were excluded. NAFLD was diagnosed based on pediatric diagnostic criteria in obese children with liver steatosis in ultrasound (US) as well as elevated alanine transaminase (ALT) serum activity after exclusion of other major liver diseases listed before. Ultrasonography was used as a screening method and for qualitative assessment of the steatosis degree (graded according to Saverymuttu scale). Advanced steatosis was defined as a score $>1$. The total intrahepatic lipid content (TILC) was assessed by magnetic resonance proton spectroscopy ('HMRS) which is the most accurate technique for assessment of ectopic fat accumulation. Fasting serum concentration of ceramides was measured in 62 children.
\end{abstract}

Results: NAFLD was diagnosed in 31 children. Significant, positive correlation was found between total serum concentration of ceramides and insulin $(r=0.3, p=0.02)$ and HOMA-IR $(r=0.28, p=0.03)$. Total ceramide concentration as well as specific fatty acid-ceramides (FA-ceramides) concentrations, namely: myristic, palmitic, palmitoleic, stearic, oleic, behenic and lignoceric were significantly higher $(p=0.004, p=0.003, p=0.007, p<0.001, p=0.035, p=0.008$, $p=0.003, p=0.006$, respectively) in children with NAFLD compared to controls $(n=14)$. Moreover, children with NAFLD had significantly higher activity of ALT $(p<0.001)$ and GGT $(p<0.001)$, HOMA-IR $(p=0.04)$, BMI $(p=0$. 046), waist circumference $(p=0.01)$ steatosis grade in ultrasound $(p<0.001)$ and TILC in ${ }^{1} H M R S(p<0.001)$ compared to children without NAFLD. We did not find significant differences in total and FA-ceramide species concentrations between children with mild (grade 1) and advanced liver steatosis in ultrasonography (grade 2-3).

Conclusion: Elevated ceramide concentrations in obese patients together with their significant correlation with insulin resistance parameters suggest their association with molecular pathways involved in insulin signaling impairment known to be strongly linked to pathogenesis of non-alcoholic fatty liver disease.

Keywords: Obesity, NAFLD, Ceramides, Fatty liver, Magnetic resonance proton spectroscopy

\footnotetext{
* Correspondence: nwasilewska@interia.pl

'Department of Pediatrics, Gastroenterology, Hepatology, Nutrition and

Allergology, Medical University of Bialystok, ul. Waszyngtona 17, 15-274

Białystok, Poland

Full list of author information is available at the end of the article
}

(c) The Author(s). 2018 Open Access This article is distributed under the terms of the Creative Commons Attribution 4.0 International License (http://creativecommons.org/licenses/by/4.0/), which permits unrestricted use, distribution, and reproduction in any medium, provided you give appropriate credit to the original author(s) and the source, provide a link to the Creative Commons license, and indicate if changes were made. The Creative Commons Public Domain Dedication waiver (http://creativecommons.org/publicdomain/zero/1.0/) applies to the data made available in this article, unless otherwise stated. 


\section{Background}

Childhood obesity caused mostly by sedentary lifestyle and hypercaloric diet has become a major worldwide health problem [1]. The global prevalence of obesity among children aged 5-19 is estimated to be $5.6 \%$ in girls and $7.8 \%$ in boys [2]. In Poland, the prevalence of overweight and obesity among children aged 718 years is reported to be over $14 \%$ in girls and over $18 \%$ in boys [3]. Following the current epidemic of obesity, the incidence of nonalcoholic fatty liver disease (NAFLD) has risen as well, making it a most common liver pathology worldwide. [4-6]. Certain features of metabolic syndrome such as visceral obesity, type 2 diabetes, hypertension and cardiovascular disease are the most common comorbidities of NAFLD. However NAFLD connection to metabolic syndrome is complex and bi-directional - the cause-and-effect relationship between them is still at the debate [7-9]. The term NAFLD has been applied to different degrees of liver steatosis - it includes changes from simple fat accumulation to steatohepatitis and fibrosis. NAFLD pathogenesis has not yet been fully established. A growing body of evidence suggest 'multiple hits hypothesis' based on assumption that lipid accumulation accompanied by insulin resistance increase susceptibility to liver damage by multiple factors such as lipotoxicity, adipocytokines, gut - derived endotoxin, endoplasmic reticulum stress and others [10]. Lipotoxicity, a process involving lipid induced oxidative stress, inflammation and cell death is among most commonly investigated mechanisms of liver injury. It has been recently established that not only the quantity but also quality of accumulated lipids plays a major role in NAFLD pathogenesis. A growing body of evidence suggest that it is the type of lipids, their relative amount and interplay that is of central importance in the process of lipotoxicity [11, 12]. Therefore the contribution of certain lipid species to NAFLD pathogenesis is commonly discussed. Although triglycerides represent a major class of accumulated lipids responsible for steatosis, they have been considered protective against cell injury [13]. Sphingolipids are another group of lipids that play significant role in cell homeostasis. They may serve as structural and signaling molecules or be transported to plasma lipoproteins. Many studies suggest their important role in metabolic syndrome. Altered sphingolipids metabolism has been found in obesity, type 2 diabetes, atherosclerosis and cardiovascular disease [14]. A recent review on the literature indicates that amongst sphingolipids ceramides (CER) may be the main lipid species involved in lipotoxicity during NAFLD [15, 16]. They have been also identified, alongside diacylglycerols as major mediator of lipid - induced insulin resistance and other cellular processes strongly linked to NAFLD - fat accumulation, oxidative stress, inflammation and cell death [17-20]. Some studies suggest that certain FA-ceramide species may be more pathogenic than the other ones. Ceramides containing long side chains such as palmitic (CER 16:0) and stearic (CER 18:0) are suspected to be major molecules involved in insulin resistance and hepatic steatosis [21, 22]. In recent years there has been considerable interest in the role of different lipid species in the pathogenesis of metabolic diseases however our knowledge on this subject in pediatric patients is based on limited data. Therefore the main aim of our research was to evaluate the serum ceramides concentration and their correlation with the steatosis degree, anthropometric measurements, insulin resistance and other biochemical parameters in obese children with NAFLD. The purpose of this analysis is to determine if ceramides are related to NAFLD pathogenesis and if they correlate with disease severity. Another objective of the study was to investigate if they may serve as a possible molecular mediator of lipid-induced hepatic insulin resistance.

\section{Methods}

The prospective study included 80 children (60 males and 20 females) at median age 12 (7-17 years) with body mass index $(\mathrm{BMI})>95$ th percentile admitted to our department to diagnose initially suspected liver disease (elevated serum alanine aminotransferase (ALT) activity and/or ultrasonographic liver brightness and/or hepatomegaly). Patients with viral hepatitis ( $\mathrm{HCV}, \mathrm{HBV}, \mathrm{CMV})$, autoimmune $(\mathrm{AIH})$, toxic (drug induced liver injury), selected metabolic liver diseases (Wilson's disease, alfa-1-antitrypsin deficiency cystic fibrosis) and coeliac disease were excluded. Written informed consent was obtained from parents and all participants older than 16 years old. Reference group included 15 children with normal BMI $(<85$ th percentile), without any somatic organ pathology at similar age and sex. The protocol was approved by the Bioethics Committee of the Medical University of Bialystok in accordance with the Declaration of Helsinki. All children underwent anthropometric measurements, their BMI was calculated and the corresponding percentile for their age and sex was established using OLAF growth charts [23]. The laboratory tests included activity of ALT, gamma glutamyltransferase (GGT), lipid profile (total cholesterol, lipoprotein-HDL and LDL, triglycerides) and insulin resistance indicators (glucose and insulin levels, calculated homeostasis model assessment-estimated insulin resistance (HOMA-IR) [24]). Ultrasonography was performed as a screening for liver steatosis in the beginning of the diagnostic process. The 
qualitative assessment of the degree of liver steatosis (graded according to Saverymuttu scale [25] was performed in a blinded fashion by ultrasonography performed by one radiologist. Advanced steatosis was defined as a score $>1$. Nonalcoholic fatty liver disease was diagnosed based on criteria proposed by ESPGHAN Hepatology Commitee on diagnosing NAFLD in children. In pediatric clinical practice NAFLD diagnosis is suggested by finding liver steatosis in ultrasonoghraphy and elevated serum alanine transaminase activity in obese children with exclusion of other major causes of liver disease listed earlier as exclusion criteria [26]. The total intrahepatic lipid content (TILC) was assessed in relative units (r.u) in comparison to the unsuppressed water signal by magnetic resonance proton spectroscopy ( $\left.{ }^{1} \mathrm{HMRS}\right)$, which is acknowledged method for accurate evaluation of ectopic fat accumulation [27]. ${ }^{1} \mathrm{HMR}$ spectroscopy was performed with a $1.5 \mathrm{~T}$ scanner (Picker Eclipse) and with PRESS sequencing. Fasting serum concentration of ceramides was measured in 62 children.

In order to quantify ceramides, a small volume of the chloroform phase containing lipids was carefully injected to a tube with N-palmitoyl-D-erythro-sphingosine (C17 base) as an internal standard. Thin-layer chromatography silica plates (Kieselgel 60, $0.22 \mathrm{~mm}$, Merck, Darmstadt, Germany) with a heptane:isopropyl ether:acetic acid (60:40:3, $\mathrm{vol} / \mathrm{vol} / \mathrm{vol})$ resolving solution were then used to separate lipid fractions. To visualize lipid bands, plates were sprayed with $0.2 \%$ solution of $3^{\prime} 7^{\prime}$-dichlorofluorescin in methanol. Utraviolet light was then used to identify the bands using standards. The gel bands were removed from the plate, placed in the screw tubes and transmethylated with BF3/methanol. Next, HewlettPackard 5890 Series II gas chromatograph with Varian CPSIL capillary column (50 m $0.25 \mathrm{~mm}$ internal diameter) and flame-ionization detector (Agilent Technologies, Santa Clara, CA) was used to analyze fatty acid methyl esters (FAMEs) dissolved in hexane. The temperature of injector and detector was set at $250 \mathrm{C}$. The oven temperature was increased linearly from 160 to $225 \mathrm{C}$ at a rate of $5 \mathrm{C} / \mathrm{min}$. The long-chain fatty acids were quantified according to the retention times of standards. Total content of CER was estimated as the total of the particular fatty acid species of the assessed fraction and it was expressed in nanomoles per milliliter of the serum. Statistical analysis was performed using Statistica 12.0 software. The serum concentrations of biochemical parameters were expressed as median; 25-75 quartile (Q1-Q3). Mann-Whitney two-sample test was used for comparisons of nonparametric data. Correlations were analyzed by the Spearman rank-correlation test for non-parametric data and by the Pearson method for parametric data. The tests were considered statistically significant at $p<0.05$.

\section{Results}

The research included eighty obese patients (60 males and 20 females) as a study group and 15 children with normal BMI without any somatic organ pathology as a reference group. Median BMI was 27.79 (25.85-31.85) kg/m². Nonalcoholic fatty liver disease was diagnosed in 31 children (38.75\%). Mild steatosis was diagnosed in 49 (61.25\%) and advanced in 31 (38.75\%) children respectively. Insulin resistance was observed in 62 individuals (77.50\%). Characteristics of the study group is presented in the Table 1.

Patients with the diagnosis of NAFLD had significantly higher waist circumference $(p<0.05)$, GGT activity $(p<0.001)$, HOMA-IR $(p<0.05)$ and liver lipid content in ${ }^{1}$ HMRS $(p<0.001)$ when compared to the rest of obese patients. Similar differences were observed in comparison between NAFLD patients and non-hepatopathic obese children (no steatosis or ALT elevation) (Table 2).

Total ceramide concentration was significantly higher in the study group than in the reference group $(p<$ 0.001). Likewise, group of children with NAFLD reported significantly higher total ceramide concentration than the reference group $(p=0.004)$ (Fig. 1). Selected FA-ceramide concentrations assessed in the study group in comparison to reference group are presented in Table 3. Further analysis has shown that patients with NAFLD had significantly higher concentration of certain FA-ceramides when compared to reference group (Table 4).

Significant, positive correlation was found between serum total ceramide concentration and insulin concentration $(R=0.286)$ and HOMA-IR index $(R=0.278)$ (Fig. 2).

It did not correlate with transaminases, triglycerides, total cholesterol, HDL - cholesterol, LDL - cholesterol nor the amount of lipids measured in ${ }^{1}$ HMRS. Moreover

Table 1 Characteristics of the study group $(n=80)$

\begin{tabular}{ll}
\hline Parameter & Median (Q1-Q3 \\
\hline Age (years) & $12(11-15)$ \\
BMI $\left(\mathrm{kg} / \mathrm{m}^{2}\right)$ & $27.8(25.9-31.9)$ \\
Waist $(\mathrm{cm})$ & $94(90-104)$ \\
ALT (IU/l) & $35.5(21.5-59.0)$ \\
GGT (IU/l) & $21(15.5-30.0)$ \\
Cholesterol (mg/dl) & $177.5(146.0-191.5)$ \\
HDL-cholesterol (mg/dl) & $47.5(39-54)$ \\
LDL - cholesterol (mg/dl) & $99.5(79.5-121.0)$ \\
Triglycerides (TG) (mg/dl) & $107.5(84.0-152.5)$ \\
Glucose (mg/dl) & $92.0(85.5-96.0)$ \\
Insulin ( $\mu l \mathrm{l} / \mathrm{ml})$ & $15.0(11.7-19.9)$ \\
HOMA - IR & $3.5(2.6-4.5)$ \\
TILC (r.u) & $107(58-180)$ \\
\hline
\end{tabular}


Table 2 Comparison of selected parameters between the group of NAFLD patients and non-hepatopathic obese patients

\begin{tabular}{|c|c|c|c|}
\hline Parameter & $\begin{array}{l}\text { NAFLD group }(n=31) \\
\text { Median }(\mathrm{Q} 1-\mathrm{Q} 3)\end{array}$ & $\begin{array}{l}\text { Non - hepatopathic obese }(n=12) \\
\text { Median (Q1-Q3) }\end{array}$ & $P$ \\
\hline Age (years) & $14(11-16)$ & $12(10.5-14)$ & ns \\
\hline BMI $\left(\mathrm{kg} / \mathrm{m}^{2}\right)$ & $28.1(26.6-33.5)$ & $26.2(25.3-29.5)$ & 0.035 \\
\hline Waist (cm) & $98(94-107)$ & $87(82-94)$ & 0.004 \\
\hline ALT (IU/I) & $64(51-104)$ & $18(15-21)$ & $<0.001$ \\
\hline GGT (IU/I) & $30(22-45)$ & $15.0(13.0-18.5)$ & $<0.001$ \\
\hline Cholesterol (mg/dl) & $181(159-216)$ & 162.5 (139.5-182.0) & ns \\
\hline HDL-cholesterol (mg/dl) & $45(40-51)$ & $50.5(41-55.5)$ & ns \\
\hline LDL - cholesterol (mg/dl) & $105(80-145)$ & $89.5(74.0-105.5)$ & ns \\
\hline Triglycerides (TG) (mg/dl) & $140(85-167)$ & $87.5(73.0-121.0)$ & 0.037 \\
\hline Glucose (mg/dl) & 93 (84-99) & $91(86-96)$ & ns \\
\hline Insulin ( $\mu \mid \cup / m l)$ & $17.4(13.8-21.0)$ & $14.8(10.3-17.7)$ & ns \\
\hline HOMA - IR & $3.9(2.8-4.8)$ & $3.4(2.3-3.8)$ & ns \\
\hline TILC (r.u) & $172(120-216)$ & $29(17-58)$ & $<0.001$ \\
\hline
\end{tabular}

CER lignoceric was positively correlated with HOMA-IR and insulin $(R=0.276, R=265$ respectively).

\section{Discussion}

In the current research we assessed the concentration of circulating ceramides (total and FA-ceramide species) in obese pediatric patients in reference to their clinical and laboratory data. The results of this study show significantly higher total serum CER concentration in obese patients as well as patients with NAFLD when compared to controls. Moreover, we observed significantly higher levels of certain ceramides (C14:0, C16:0, C16:1, C18:0, C18:1, C22:0, C24:0) in the serum of obese children and patients with NAFLD in comparison to reference group. Another important finding was that total ceramide concentration was positively correlated with HOMA-IR and insulin levels.

In accordance with the present results, previous studies on humans and animals have demonstrated significantly increased total serum CER concentrations and specific FA - ceramides in obese populations [17, 18, 28]. However, among these studies, Haus et al. and Lopez et al. reported higher ceramide concentrations in obese populations with type 2 diabetes, disease known to be independently related to increased serum CER levels. On the other hand, study by Majumdar et al. did not report significant elevation of neither total serum CER nor specific CER in

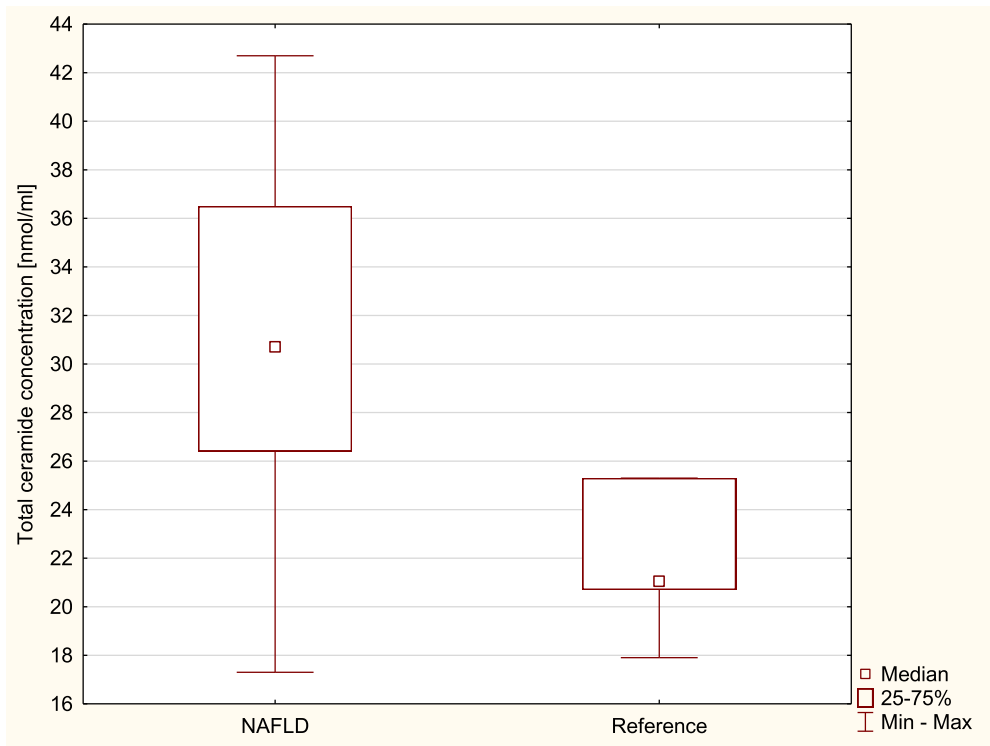

Fig. 1 Total ceramide concentrations in serum of the patients with NAFLD (NAFLD) and reference group (Reference) 
Table 3 Differences between serum FA-ceramide (CER) ( $\mathrm{nmol} / \mathrm{ml}$ ) concentrations in study group and reference group

\begin{tabular}{llll}
\hline Ceramide (FA) & $\begin{array}{l}\text { Study group } \\
\text { M (Q1-Q3) }\end{array}$ & $\begin{array}{l}\text { Reference group } \\
\text { M (Q1-Q3) }\end{array}$ & $p$ value \\
\hline CER myristic (C14:0) & $2.1(1.4-4.3)$ & $0.9(0.7-1.2)$ & $<0.001$ \\
CER palmitic (C16:0) & $8.6(6.7-10.4)$ & $5.2(4.8-6.8)$ & $<0.001$ \\
CER palmitoleic (C16:1) & $1.0(0.8-1.3)$ & $0.6(0.5-0.8)$ & $<0.001$ \\
CER stearic (C18:0) & $5.6(4.5-7.6)$ & $4.0(3.6-4.5)$ & $<0.005$ \\
CER oleic (C18:1) & $2.8(2.4-3.6)$ & $2.1(1.9-2.8)$ & $<0.005$ \\
CER linoleic (C18:2) & $0.3(0.0-1.0)$ & $0.2(0.0-0.7)$ & $n s$ \\
CER arachidic (C20:0) & $0.5(0.4-0.6)$ & $0.4(0.4-0.5)$ & $\mathrm{ns}$ \\
CER linolenic (C18:3) & $0.3(0.2-0.3)$ & $0.2(0.2-0.3)$ & $\mathrm{ns}$ \\
CER behenic (C22:0) & $1.5(1.3-1.7)$ & $1.3(1.1-1.4)$ & $<0.01$ \\
CER arachidonic (C20:4) & $0.5(0.4-0.7)$ & $0.5(0.4-0.7)$ & $\mathrm{ns}$ \\
CER lignoceric (C24:0) & $3.3(2.8-4.1)$ & $3.0(2.6-3.3)$ & $<0.05$ \\
CER eicosapentaenoic (C20:5) & $0.8(0.7-0.9)$ & $0.8(0.7-0.9)$ & $\mathrm{ns}$ \\
CER nervinic (C24:1) & $2.3(2.0-2.6)$ & $2.0(1.9-2.2)$ & $\mathrm{ns}$ \\
CER docosahexaenoic (C22:6) & $0.7(0.6-0.9)$ & $0.7(0.6-0.8)$ & $\mathrm{ns}$ \\
\hline
\end{tabular}

the group of overweight children in comparison to lean controls [29]. This discrepancy could be attributed to smaller study group and differences in inclusion criteria (overweight vs. obese). Previous studies on ceramide concentrations in NAFLD individuals performed on humans and mice report findings which support the results of our study. Significantly higher total CER concentration as well as a major increase in C16:0, C22:0, C24:0, C24:1 in NAFLD mice was noticed by Kasumov et al. [30]. Recent data suggest a major role of C16-ceramides overaccumulation in metabolic diseases, mainly due to impaired hepatic

Table 4 Differences between serum FA-ceramide (CER) ( $\mathrm{nmol} / \mathrm{ml}$ ) concentrations in children with NAFLD and reference group

\begin{tabular}{llll}
\hline Ceramide (FA) & NAFLD group & $\begin{array}{l}\text { Reference group } \\
\text { M (Q1-Q3) }\end{array}$ & M (Q1-Q3) \\
\hline CER myristic (C14:0) & $1.9(1.3-2.3)$ & $0.9(0.7-1.2)$ & $<0.005$ \\
CER palmitic (C16:0) & $7.8(6.5-10.0)$ & $5.2(4.8-6.8)$ & $<0.01$ \\
CER palmitoleic (C16:1) & $0.9(0.7-1.1)$ & $0.6(0.5-0.8)$ & $<0.001$ \\
CER stearic (C18:0) & $5.2(4.3-6.8)$ & $4.0(3.6-4.5)$ & $<0.05$ \\
CER oleic (C18:1) & $2.9(2.4-3.7)$ & $2.1(1.9-2.8)$ & $<0.05$ \\
CER linoleic (C18:2) & $0.5(0.1-1.1)$ & $0.2(0.0-0.7)$ & $\mathrm{ns}$ \\
CER arachidic (C20:0) & $0.5(0.4-0.6)$ & $0.4(0.4-0.5)$ & $\mathrm{ns}$ \\
CER linolenic (C18:3) & $0.3(0.2-0.4)$ & $0.2(0.2-0.3)$ & $\mathrm{ns}$ \\
CER behenic (C22:0) & $1.6(1.4-1.7)$ & $1.3(1.1-1.4)$ & $<0.005$ \\
CER arachidonic (C20:4) & $0.6(0.4-0.7)$ & $0.5(0.4-0.7)$ & $\mathrm{ns}$ \\
CER lignoceric (C24:0) & $3.7(3.0-4.1)$ & $3.0(2.6-3.3)$ & $<0.01$ \\
CER eicosapentaenoic (C20:5) & $0.7(0.7-0.9)$ & $0.8(0.7-0.9)$ & $\mathrm{ns}$ \\
CER nervinic (C24:1) & $2.3(2.0-2.5)$ & $2.0(1.9-2.2)$ & $\mathrm{ns}$ \\
CER docosahexaenoic (C22:6) & $0.7(0.6-0.8)$ & $0.7(0.6-0.8)$ & $\mathrm{ns}$ \\
\hline
\end{tabular}

fatty acid oxidation. It has been hypothesized that long-chain FA-ceramides such as C16-ceramide increase liver susceptibility for diet-induced steatohepatitis and insulin resistance in obese individuals [21,31]. Increased levels of C16:0 ceramide in serum of NAFLD patients seem to be consistent with these studies. Luukkonen et al. presented an elegant study on liver ceramide levels in adults with nonalcoholic fatty liver disease and provided an important evidence that ceramides may play a major role in triggering insulin resistance in individuals with NAFLD. In this study patients were divided into two groups - 'metabolic NAFLD' defined by insulin - resistance and PNPLA3 - associated NAFLD. The analysis revealed higher levels of C16:0, C18:0, C19:0, C20:0, C22:0, $\mathrm{C} 24: 1$ dihydroceramides in the group of adults with 'metabolic NAFLD' [22]. This study points out up that composition of hepatic fatty acids lipidome impacts patient's phenotype, unfortunately no correlation with plasma ceramides was done. Recent review on lipid accumulation in pediatric patients with NAFLD also strongly highlights the role of hepatic fat composition in progression of the disease and suggests some possible novel therapeutic targets [12]. Positive correlation between total ceramide concentration and insulin resistance parameters reported in this study is consistent with other research performed on adult and pediatric patients $[17,18,22]$. Taken together these studies provide important insights into the possible link of ceramides to pathogenesis of nonalcoholic fatty liver disease.

In the literature there are only few studies on serum ceramide concentration in pediatric population. This is a first study conducted in obese children with nonalcoholic fatty liver disease investigating serum ceramide concentration. The novelty of these findings is the main strength of our study. However there are some limitations to our study. We did not use histological examination in the diagnosis of NAFLD. According to ESPGHAN Hepatology Committee liver biopsy is required for definitive diagnosis of NAFLD, but is not proposed in screening [32]. This procedure is an 'imperfect' gold standard - it is invasive, there is a considerable variability of sample and it provides only static information. However it needs to performed in some unclear cases to distinguish NAFLD from other liver diseases. Other indications for liver biopsy include suspected advanced disease, young age, hepatosplenomegaly, considerable increase in ALT activity and before pharmacological intervention. Our patients did not meet these criteria, therefore liver biopsy was not performed [33]. Another limitation of our study is a use of indirect method of insulin resistance assessment (HOMA-IR), while the euglycemic hyperinsulinemic clamp technique is considered to be a golden standard for insulin sensitivity evaluation [34]. 


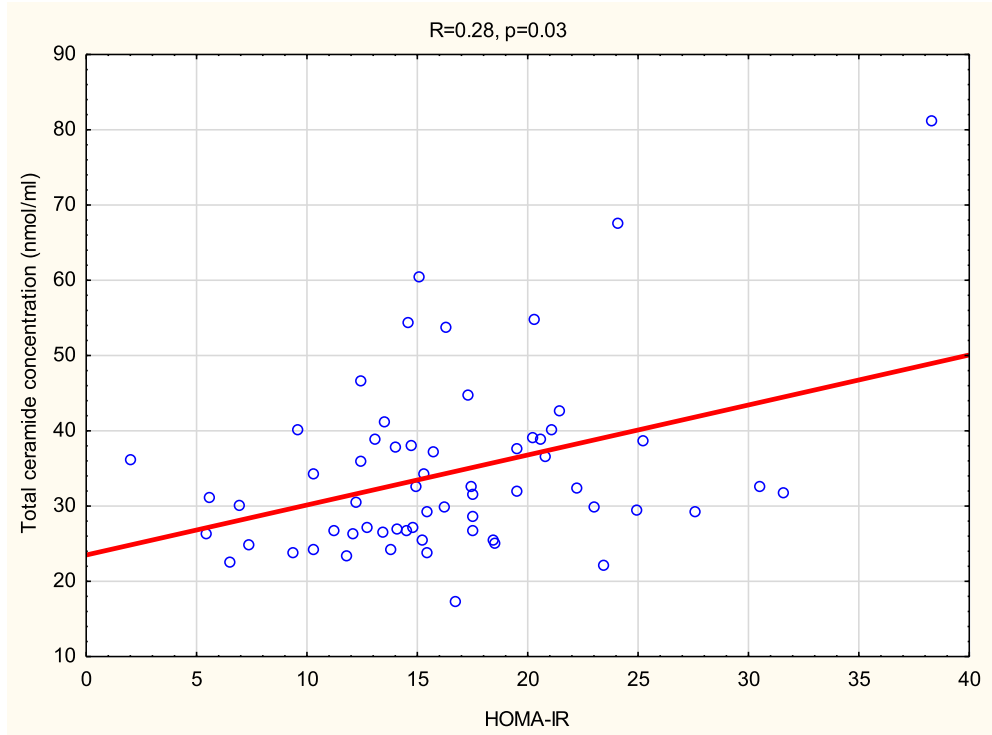

Fig. 2 Correlation between serum total ceramide concentration and HOMA-IR index

\section{Conclusion}

To conclude, the purpose of the current study was to determine plasma ceramide concentration in obese adolescents. Elevated ceramide concentrations in obese patients together with their significant correlation with insulin resistance parameters suggest their association with molecular pathways involved in insulin signaling impairment known to be strongly linked to pathogenesis of non-alcoholic fatty liver disease. Further work is needed to determine exact role of ceramides in the pathogenesis of this disease but in the future it may serve as clinical tool and another potential therapeutic target.

\section{Abbreviations \\ 'HMRS: Magnetic resonance proton spectroscopy; AlH: Autoimmune hepatitis; ALT: Alanine transaminase; BMI: Body mass index; CER: Ceramide; CMV: Cytomegalovirus; FA-Ceramide: Fatty acid - ceramide; GGT: Gamma glutamyltransferase; HBV: Hepatitis B virus; HCV: Hepatitis C virus; HDL: High- density lipoprotein; HOMA - IR: Homeostasis model assessment-estimated in- sulin resistance; LDL: Low-density lipoprotein; NAFLD: Nonalcoholic fatty liver disease; TILC: Total intrahepatic lipid content; US: Ultrasound}

\section{Funding}

The study was financially supported by Grants from Medical University of Bialystok, Poland, N/ST/ZB/15/001/1143 and N/ST/ZB/15/003/1143.

\section{Availability of data and materials}

The datasets used and/or analysed during the current study are available from the corresponding author on reasonable request.

\section{Authors' contributions}

$\mathrm{DL}$ and $\mathrm{AC}$ designed and supervised the project. $\mathrm{DL}$ and $\mathrm{ABC}$ acquired the data for further analysis. ET and MW performed radiological examinations, collected data and helped with data analysis and interpretation. EHS and AC performed biochemical experiments to quantify ceramides concentrations and contributed to the interpretation of the results. NW processed the experimental data, performed the analysis, drafted the manuscript and designed the figures and Tables. NW wrote the manuscript with input from all authors. All authors provided critical feedback and helped shape the research, analysis and manuscript. All authors read and approved the final manuscript.

Ethics approval and consent to participate

The protocol was approved by the Bioethics Committee of the Medical University of Bialystok in accordance with the Declaration of Helsinki.

\section{Consent for publication}

Not applicable

\section{Competing interests}

The authors declare that they have no competing interests.

\section{Publisher's Note}

Springer Nature remains neutral with regard to jurisdictional claims in published maps and institutional affiliations.

\section{Author details}

'Department of Pediatrics, Gastroenterology, Hepatology, Nutrition and Allergology, Medical University of Bialystok, ul. Waszyngtona 17, 15-274 Białystok, Poland. ${ }^{2}$ Department of Physiology, Medical University of Bialystok, Bialystok, Poland. ${ }^{3}$ Department of Radiology, Medical University of Bialystok, Bialystok, Poland. " ${ }^{4}$ Department of Radiology, University Teaching Children's Hospital, Bialystok, Poland.

Received: 16 July 2018 Accepted: 28 August 2018

Published online: 12 September 2018

\section{References}

1. Ng M, Fleming T, Robinson M, Thomson B, Graetz N, Margono C, et al. Global, regional, and national prevalence of overweight and obesity in children and adults during 1980-2013: a systematic analysis for the global burden of disease study 2013. Lancet. 2014;384(9945):766-81.

2. (NCD-RisC) NRFC. Worldwide trends in body-mass index, underweight, overweight, and obesity from 1975 to 2016: a pooled analysis of 2416 population-based measurement studies in 128.9 million children, adolescents, and adults. Lancet. 2017;390(10113):2627-42.

3. Kułaga Z, Grajda A, Gurzkowska B, Wojtyło MA, Góźdź M, Litwin MS. The prevalence of overweight and obesity among polish school- aged children and adolescents. Przegl Epidemiol. 2016;70(4):641-51.

4. Brunt EM, Wong WW, Nobili V, Day CP, Sookoian S, Maher JJ, et al. Nonalcoholic fatty liver disease. Nat Rev Dis Primers. 2015;1:15080. 
5. Welsh JA, Karpen S, Vos MB. Increasing prevalence of nonalcoholic fatty liver disease among United States adolescents, 1988-1994 to 2007-2010. J Pediatr. 2013;162(3):496-500.e1.

6. Younossi ZM, Koenig AB, Abdelatif D, Fazel Y, Henry L, Wymer M. Global epidemiology of nonalcoholic fatty liver disease-meta-analytic assessment of prevalence, incidence, and outcomes. Hepatology. 2016;64(1):73-84.

7. Corte CD, Ferrari F, Villani A, Nobili V. Epidemiology and Natural history of NAFLD. J Med Biochemist. 2015;34(1):13-7.

8. George J, Anstee Q, Ratziu V, Sanyal A. NAFLD: the evolving landscape. J Hepatol. 2018;68(2):227-9.

9. Lonardo A, Nascimbeni F, Mantovani A, Targher G. Hypertension, diabetes, atherosclerosis and NASH: cause or consequence? J Hepatol. 2018;68(2): 335-52.

10. Petta S, Gastaldelli A, Rebelos E, Bugianesi E, Messa P, Miele L, et al. Pathophysiology of non alcoholic fatty liver disease. Int J Mol Sci. 2016;17: (12):2082-107

11. Marra F, Svegliati-Baroni G. Lipotoxicity and the gut-liver axis in NASH pathogenesis. J Hepatol. 2018;68(2):280-95.

12. Mann JP, Feldstein AE, Nobili V. Update on lipid species and paediatric nonalcoholic fatty liver disease. Curr Opin Clin Nutr Metab Care. 2017; 20(2):110-6.

13. Yamaguchi K, Yang L, McCall S, Huang J, Yu XX, Pandey SK, et al. Inhibiting triglyceride synthesis improves hepatic steatosis but exacerbates liver damage and fibrosis in obese mice with nonalcoholic steatohepatitis. Hepatology. 2007;45(6):1366-74

14. Meikle PJ, Summers SA. Sphingolipids and phospholipids in insulin resistance and related metabolic disorders. Nat Rev Endocrinol. 2017;13(2):79-91.

15. Chaurasia B, Summers SA. Ceramides - Lipotoxic inducers of metabolic disorders. Trends Endocrinol Metab. 2015;26(10):538-50.

16. Kurek K, Piotrowska DM, Wiesiołek-Kurek P, Łukaszuk B, Chabowski A, Górski J, et al. Inhibition of ceramide de novo synthesis reduces liver lipid accumulation in rats with nonalcoholic fatty liver disease. Liver Int. 2014;34(7):1074-83.

17. Lopez X, Goldfine AB, Holland WL, Gordillo R, Scherer PE. Plasma ceramides are elevated in female children and adolescents with type 2 diabetes. J Pediatr Endocrinol Metab. 2013;26(9-10):995-8.

18. Haus JM, Kashyap SR, Kasumov T, Zhang R, Kelly KR, Defronzo RA, et al. Plasma ceramides are elevated in obese subjects with type 2 diabetes and correlate with the severity of insulin resistance. Diabetes. 2009;58(2):337-43.

19. Ilan Y. Compounds of the sphingomyelin-ceramide-glycosphingolipid pathways as secondary messenger molecules: new targets for novel therapies for fatty liver disease and insulin resistance. Am J Physiol Gastrointest Liver Physiol. 2016; 310(11):G1102-17.

20. Petersen MC, Madiraju AK, Gassaway BM, Marcel M, Nasiri AR, Butrico G, et al. Insulin receptor Thr1160 phosphorylation mediates lipid-induced hepatic insulin resistance. J Clin Invest. 2016;126(11):4361-71.

21. Turpin SM, Nicholls HT, Willmes DM, Mourier A, Brodesser S, Wunderlich CM, et al. Obesity-induced CerS6-dependent C16:0 ceramide production promotes weight gain and glucose intolerance. Cell Metab. 2014;20(4):678-86.

22. Luukkonen PK, Zhou $Y$, Sädevirta $S$, Leivonen $M$, Arola J, Orešič $M$, et al. Hepatic ceramides dissociate steatosis and insulin resistance in patients with non-alcoholic fatty liver disease. J Hepatol. 2016;64(5):1167-75.

23. Kulaga Z, Litwin M, Tkaczyk M, Rózdzyńska A, Barwicka K, Grajda A, et al. The height-, weight-, and BMl-for-age of polish school-aged children and adolescents relative to international and local growth references. BMC Public Health. 2010;10:109.

24. Matthews DR, Hosker JP, Rudenski AS, Naylor BA, Treacher DF, Turner RC. Homeostasis model assessment: insulin resistance and beta-cell function from fasting plasma glucose and insulin concentrations in man. Diabetologia. 1985;28(7):412-9.

25. Saverymuttu SH, Joseph AE, Maxwell JD. Ultrasound scanning in the detection of hepatic fibrosis and steatosis. Br Med J (Clin Res Ed). 1986:292(6512):13-5.

26. Vajro P, Lenta S, Socha P, Dhawan A, McKiernan P, Baumann U, et al. Diagnosis of nonalcoholic fatty liver disease in children and adolescents: position paper of the ESPGHAN hepatology committee. J Pediatr Gastroenterol Nutr. 2012. 54(5):700-13.

27. Bohte $A E$, van Werven JR, Bipat S, Stoker J. The diagnostic accuracy of US, $C T, M R I$ and $1 \mathrm{H}-\mathrm{MRS}$ for the evaluation of hepatic steatosis compared with liver biopsy: a meta-analysis. Eur Radiol. 2011;21(1):87-97.

28. Samad F, Hester KD, Yang G, Hannun YA, Bielawski J. Altered adipose and plasma sphingolipid metabolism in obesity: a potential mechanism for cardiovascular and metabolic risk. Diabetes. 2006;55(9):2579-87.
29. Majumdar I, Mastrandrea LD. Serum sphingolipids and inflammatory mediators in adolescents at risk for metabolic syndrome. Endocrine. 2012;41(3):442-9.

30. Kasumov T, Li L, Li M, Gulshan K, Kirwan JP, Liu X, et al. Ceramide as a mediator of non-alcoholic fatty liver disease and associated atherosclerosis. PLoS One. 2015;10(5):e0126910.

31. Raichur S, Wang ST, Chan PW, Li Y, Ching J, Chaurasia B, et al. CerS2 Haploinsufficiency inhibits $\beta$-oxidation and confers susceptibility to dietinduced steatohepatitis and insulin resistance. Cell Metab. 2014;20(5):919.

32. Dezsőfi A, Baumann U, Dhawan A, Durmaz O, Fischler B, Hadzic N, et al. Liver biopsy in children: position paper of the ESPGHAN hepatology committee. J Pediatr Gastroenterol Nutr. 2015;60(3):408-20

33. Della Corte C, Vajro P, Socha P, Nobili V. Pediatric non-alcoholic fatty liver disease: recent advances. Clin Res Hepatol Gastroenterol. 2014;38(4):419-22.

34. Straczkowski M, Stepień A, Kowalska I, Kinalska I. Comparison of simple indices of insulin sensitivity using the euglycemic hyperinsulinemic clamp technique. Med Sci Monit. 2004;10(8):CR480-4.
Ready to submit your research? Choose BMC and benefit from:

- fast, convenient online submission

- thorough peer review by experienced researchers in your field

- rapid publication on acceptance

- support for research data, including large and complex data types

- gold Open Access which fosters wider collaboration and increased citations

- maximum visibility for your research: over $100 \mathrm{M}$ website views per year

At $\mathrm{BMC}$, research is always in progress.

Learn more biomedcentral.com/submissions 